\title{
Muscular activity in different locomotion plans with the use of various shoes types and barefoot
}

\author{
Geraldo Fabiano de Souza Moraes ${ }^{\mathrm{a},}$, , Davidson Passos Mendes ${ }^{\mathrm{b}}$, Adriana Antunes Papinni ${ }^{\mathrm{c}}$ \\ ${ }^{a}$ Physiotherapist, M.Sc Rehabilitation Science, Assistent Professor at University Federal of Itajubá - Campus \\ Itabira, Itabira, Minas Gerais, Brazil.E-mail: geraldofsmoraes@gmail.com \\ ${ }^{\mathrm{b}}$ Physiotherapist, M.Sc Production Engineering Assistent Professor at University Federal of Itajubá - Campus \\ Itabira, Itabira, Minas Gerais, Brazil. E-mai: davidsonpmendes@gmail.com \\ ${ }^{c}$ Physiotherapist, B.Sc Physical Therapy E-mail: dripantunes@yahoo.com.br
}

\begin{abstract}
The objective of this study was to evaluate the electromyographyc activity of the muscles of the leg and spine associated with different types of shoes and barefoot, in the various plans of locomotion, in the situations before and after fatigue. Methods: Is a cross-sectional study, which was used a sample of convenience with 15 young women, aged between 18 and 35 years, sedentary. The examined muscles were tibialis anterior, gastrocnemius medialis and lateralis and erector spinae. The muscular activity was assessed during locomotion on flat surface, up and down stairs and in an inclined surface. The shoes were a high-heeled, small heeled, tennis, slipper and barefoot. Results: The results of electromyographyc analysis showed that the muscles of the leg had greater electromyographyc activity in the position of pre-fatigue of the lower limbs and erector spinae in the postfatigue of the lower limbs, in most of the conditions and situations. Discussion: Adaptive conditions associated with regular use of high shoes, has been widely questioned in relation to the incidence of injuries. Conclusion: In post-fatigue of the lower limbs, there was a decline in the activities of the muscles of the leg and increased recruitment of the erector muscles justified by the need for greater stability of the spine and pelvis during the locomotion of young women.
\end{abstract}

Keywords: Ergonomics, occupational risk, gait, shoes

\section{Introduction}

One of the most prevalent aspects are women with complaints of discomfort in the lumbar region caused by the use of high heeled shoe [1]. Snow and Williams [2] described that there may be a link between attitude and the present discomfort, often associated with muscular fatigue and increase the forces of reaction soil during the use of such footwear.

Esenyel et al. [3]), Hong et al. [4] and Snow and Williams [2] showed that the major changes occur in the lower limbs (LL), according to the height of the heel, occurs a significant increase in plantarflexion, changes in the orientation of bone structures of the ankle joints, metatarsal and metatarsophalangeals, changes in angles of insertion of the muscles of the foot and ankle and decrease the maximum angle of the knee during the balance and in the speed of knee's extension.

Previous work suggests that altered muscle activity will have a predictable effect on ground reaction forces. Such work has shown that the plantarflexors are the primary muscles contributing to the propul-

\footnotetext{
${ }^{*}$ Corresponding author. University of Itajubá UNIFEI. Curso de Engenharia de Saúde e Segurança. Rua Um s/nº Distrito Industrial. Itabira. Minas Gerais. Brasil. CEP 35903-081. E-mail: geraldmoraes@terra.com.br
} 
sive impulse in the last half of stance during normal, unimpaired walking $[5,6]$.

More specifically, the model developed by Neptune et al. [7] showed that the gastrocnemius medialis (GM) is the only ankle extensor that aids in swing initiation. In summary, there is little consensus regarding the functional role of the individual muscles pertinent to the swing phases of walking.

1.1. In walking, the body vaults up and over each stiff leg in an arc, analogous to an inverted pendulum. Kinetic energy in the first half of the stance phase is transformed into gravitational potential energy, which is partially recovered as the body falls forward and downward in the second half of the stance phase. As a leg strikes the ground, kinetic and gravitational potential energy is temporarily stored as elastic strain energy in muscles, tendons, and ligaments and then is nearly all recovered during the propulsive second half of the stance phase [8].

The static work is highly fatiguing and, whenever possible, should be avoided. When it is not possible, can be alleviated through changes in posture, use of comfortable shoes or through support for parts of the body in order to reduce the static contractions of the muscles [9].

Among the best resources for prevention is to choose the lowest shoes, which give greater support and balance, not forcing some areas of the body. Another good practice is regularly lengthening the muscles of the thigh and calf [10].

Today, more women are included in the labor market and the use of height heels is frequent, mainly associated with the aesthetic issue. Little has been studied on the influence of footwear in the joints of LL. Due to reports of annoyances such as pain, postural changes and deformities in the feet, it is extremely important to check what type of shoes would be more appropriate and more comfortable for everyday use.

\subsection{Objective}

The objective of this study was to evaluate the electromyographyc activity of the muscles of the leg and spine associated with different types of shoes and barefoot, in the various plans of locomotion, in the situations before and after fatigue.

\section{Methodology}

\subsection{Design of the study and sample}

This is a cross-sectional study, which was used a sample of convenience with 15 young women, aged between 18 and 35 years, sedentary. The exclusion criteria were women who had joint stiffness, pain that disable the tests, lower limbs discrepancy (over one centimeter), had the ankle sprain less than six months, have been diagnosed with ligament rupture in the ankles, have been subjected to physical therapy or surgery in the lower limbs less than six months. Obese women - Body mass index (BMI) $\geq 30$ [11], pregnant, diabetic, wear aid to march, presence of swelling of the ankle and feet, hypertrophy over two centimeters of calf and thigh. That possessed hallux valgus, bunion of the $\mathrm{V}$ metatarsus and Morton's neuroma that impossibilities the procedures. This study was approved by the Research Ethics Committee of the Centro Universitário Newton Paiva with the number of internal project 0082, in March 06, 2008.

\subsection{Instruments}

It was used the electromyography EMG System, model EMG 1600, (EMG System, Sao José dos Campos, Brazil), connected to a microcomputer with eight surface electrodes active bipolar $\mathrm{Ag} / \mathrm{AgCl}$. The test results were stored and analyzed by specific software, following the manufacturer's manual.

The electromyography (EMG) is a technique that allows the documentation of the electrical signals generated by muscle cells, which allows the analysis of muscle activity during movement or simple linked to a task. It is necessary to understand concepts relating to EMG to ensure the validity and reliability of this instrument of evaluation of studies in the field of rehabilitation and the clinical practice of physiotherapists.

The validity and accuracy of the assessment of muscular activity, through the use of EMG, are related to the process of tracking signals and correct conducting of the procedures for analysis and knowledge of possible interference during the process of collecting $[12,13]$.

It was used a flat surface with a distance of six meters, a ramp of one meter and sixty centimeters with an inclination of $16^{\circ}$ and a ladder with three steps, with height of $16 \mathrm{~cm}$ and width of $24 \mathrm{~cm}$.

\subsection{Procedures}

Initially, all volunteers were informed about the objectives and procedures of the study and then 
signed the term of free and informed consent. Then, there was a standardized assessment, done by a properly trained examiner, for identification, history and physical examination of each voluntary. On physical examination, was done the goniometry of dorsiflexion, bilaterally.

After evaluation, the voluntary made an assortment, through sealed envelope to randomization of the plans of locomotion and shoes to be used during the collection of muscular activity before and after the protocol of fatigue.

It was demarcated a distance of six meters for locomotion in the flat surface. During the analysis, was despised the two meters initial and final in order to avoid interference in the electromyography's collection during acceleration and deceleration of the walk.

An examiner done the trichotomy of the points of insertion of the electrodes following the recommendations of Cram et al. [12] and clean the place through friction with gauze and alcohol. The examined muscles were tibialis anterior (TA), gastrocnemius medialis (GM) and lateralis (GL) and erector spinae (ERET), all bilaterally. Two electrodes were placed in the region where the examiner found, by palpation, the region of greater muscle contraction [12]. The electrodes were posted at a distance of two centimeters of each. It placed an reference electrode on the right acromion as a way to standardize the measures.

After the placement of electrodes, it was observed the "silence" of the muscle, and then called up the contraction of muscles to be examined during their movements made by them in order to confirm the correct positioning of equipment. It was subsequently held a maximum voluntary contraction isometric (MVCI) of each muscle in the standardization of data and further analysis of muscular activity. The data were stored on specific software.

The muscular activity was assessed during locomotion on flat surface, up and down stairs (three steps with each $15 \mathrm{~cm}$ tall) and in an inclined surface $\left(16^{\circ}\right)$.

The shoes were: high-heeled type Chanel, small heeled type Chanel, tennis, slippers and barefoot. Were considered high-heeled shoes those with height between nine and eleven centimeters and small heeled shoe those between five and seven centimeters. For the tennis was necessary that it had a heel lifting of two to four centimeters.

Foot switch was used to determine the stages of the walk, flash sync with the electromyography in order to determine the beginning of the movement and recording the walk. A metronome was used to cadence and standardizes the walk speed to 110 steps per minute in the flat surface.

After collecting with the use of all shoes, in their movements for locomotion above, the participant was referred to a protocol of fatigue of the triceps surae, bilaterally. This protocol consisted in the participant execute movements of bending plant concentric and eccentrically, continuously, until the sensation of burning and not support more repetitions $[14,15]$.

After the implementation of this protocol, they did the same pre-fatigue measures, which, the sequence of footwear and activities were, previously randomized. All data for analysis of locomotion in the various plans, using the various footwear and barefoot, were stored in the specific software equipment for later analysis.

\subsection{Data Reduction}

For electromyographycs analysis was used the software AqDanalysis following the manufacturer's recommendations. To standardize the data was used calculation of the Root Mean Square (RMS) and calculation of the percentage of MVCI. The processing of data included electromyographycs rectification and filtering of signals. Data were collected at a frequency of $1000 \mathrm{~Hz}$, were filtered with a high pass filter of $10 \mathrm{~Hz}$ and with a low pass filter of $500 \mathrm{~Hz}$. The amplifiers had an input impedance of $2 \mu \theta$ and CMRR of $1000 \mu \theta$ and lower data acquisition at frequencies from 10 to $1000 \mathrm{~Hz}$.

\section{Statistical Analysis}

Descriptive statistics, tests of normality (ShapiroWilk) and tests of equal variance (Levene) were made for all variables, using the statistical package Statistical Package of Social Science for Windows (SPSS) as 15.0. (SPSS Inc C), Chatanoga, IL).

It was used to test Post Hoc with Scheffé's correction for multiple comparisons, in order to establish the effect of interaction of shoes.

For analysis of the influence of the situations of pre and post fatigue, in the different plans and with different shoes and barefoot, was used t Student paired samples. The level of significance was established $\alpha$ equal to $5 \%$.

\section{Results}

\subsection{Sample's Characterization}


Participated of this study 15 volunteers with ages ranging from 18 to 31 years $(23.4 \pm 3.37$ years $)$, sedentary. Of the fifteen voluntary, thirteen were right handed and two were left handed. The description of the volunteers is demonstrated in Table 1.

Table 1 Participants description

\begin{tabular}{cccc}
\hline Volunteer & $\begin{array}{c}\text { Age } \\
\text { (years) } \\
(\mathbf{2 3 , 4} \pm \mathbf{3 , 6})\end{array}$ & $\begin{array}{c}\text { Shoe Size (cm) } \\
\mathbf{( 3 6} \pm \mathbf{1 , 1 3 )}\end{array}$ & Dominance \\
\hline 1 & 22 & 36 & Right \\
2 & 21 & 36 & Right \\
3 & 23 & 35 & Right \\
4 & 18 & 36 & Right \\
5 & 20 & 37 & Left \\
6 & 21 & 36 & Right \\
7 & 22 & 33 & Right \\
8 & 22 & 36 & Left \\
9 & 25 & 37 & Right \\
10 & 23 & 38 & Right \\
11 & 24 & 37 & Right \\
12 & 31 & 36 & Right \\
13 & 30 & 36 & Right \\
14 & 22 & 35 & Right \\
15 & 27 & 36 & Right \\
\hline
\end{tabular}

4.2. Assessment of muscular activity pre and postfatigue

For the assessment of muscular activity with the use of shoes and barefoot in the various plans of locomotion in situations of pre and post fatigue were found the following significant differences for their conditions of use or not use of footwear.

In this study were analyzed the muscles right tibialis anterior (RTA), left tibialis anterior (LTA), right gastrocnemius medialis (RGM), left gastrocnemius medialis (LGM), right gastrocnemius lateralis (RGL), left gastrocnemius lateralis (LGL), right erector spinae (RERET) and left erector spinae (LERET) that had significant differences (Table 2).
Table 2

Comparison of the muscles eletromyographyc activity

\begin{tabular}{|c|c|c|c|}
\hline Muscle & Shoe & Condition & p Value \\
\hline \multirow{2}{*}{$\begin{array}{l}\text { RTA - } \\
\text { support } \\
\text { LLL }\end{array}$} & Barefoot & Ramp down & 0,001 \\
\hline & Small heeled & Flat surface & 0,010 \\
\hline \multirow{2}{*}{$\begin{array}{c}\text { LTA - } \\
\text { support } \\
\text { RLL }\end{array}$} & Barefoot & Ramp up & 0,005 \\
\hline & Small heeled & Flat surface & 0,028 \\
\hline \multirow{2}{*}{$\begin{array}{l}\text { RGM - } \\
\text { support } \\
\text { RLL }\end{array}$} & Slipper & Down stairs & 0,007 \\
\hline & Tennis shoe & Flat surface & 0,049 \\
\hline \multirow{3}{*}{$\begin{array}{l}\text { RGM - } \\
\text { support } \\
\text { LLL } \\
\text { LGM - } \\
\text { support } \\
\text { RLL }\end{array}$} & High heeled & Flat surface & 0,002 \\
\hline & Barefoot & Down stairs & 0,009 \\
\hline & High heeled & $\begin{array}{l}\text { Ramp up } \\
\text { Up stairs }\end{array}$ & $\begin{array}{l}0,039 \\
0,049\end{array}$ \\
\hline \multirow{2}{*}{$\begin{array}{l}\text { LGM - } \\
\text { support } \\
\text { LLL }\end{array}$} & Tennis shoe & Up stairs & 0,032 \\
\hline & High heeled & $\begin{array}{l}\text { Flat surface } \\
\text { Ramp down }\end{array}$ & $\begin{array}{l}0,008 \\
0,036\end{array}$ \\
\hline \multirow{3}{*}{$\begin{array}{c}\text { RGL - } \\
\text { support } \\
\text { RLL } \\
\text { LGL - } \\
\text { support } \\
\text { LLL }\end{array}$} & Barefoot & Flat surface & 0,007 \\
\hline & Barefoot & Ramp up & 0,001 \\
\hline & Small heeled & Ramp up & 0,036 \\
\hline \multirow{14}{*}{$\begin{array}{c}\text { RERET - } \\
\text { support } \\
\text { RLL }\end{array}$} & Barefoot & Up stairs & 0,011 \\
\hline & & Down stairs & 0,001 \\
\hline & Slipper & Flat surface & 0,049 \\
\hline & & Down stairs & 0,001 \\
\hline & & Ramp up & 0,001 \\
\hline & & Ramp down & 0,015 \\
\hline & Tennis shoe & Flat surface & 0,008 \\
\hline & & Down stairs & 0,022 \\
\hline & & Ramp up & 0,022 \\
\hline & & Ramp down & 0,016 \\
\hline & Small heeled & Up stairs & 0,033 \\
\hline & & Ramp up & $<0,001$ \\
\hline & High heeled & Flat surface & 0,008 \\
\hline & & Ramp down & 0,015 \\
\hline
\end{tabular}




\begin{tabular}{|c|c|c|c|}
\hline \multirow{15}{*}{$\begin{array}{l}\text { RERET - } \\
\text { support } \\
\text { LLL }\end{array}$} & Barefoot & Up stairs & 0,034 \\
\hline & & Down stairs & 0,014 \\
\hline & & Ramp down & 0,002 \\
\hline & Slipper & Flat surface & 0,002 \\
\hline & & Up stairs & 0,002 \\
\hline & & Ramp up & $<0,001$ \\
\hline & & Ramp down & 0,002 \\
\hline & Tennis shoe & Flat surface & 0,016 \\
\hline & & Down stairs & 0,001 \\
\hline & & Ramp up & $<0,001$ \\
\hline & Small heeled & Down stairs & 0,001 \\
\hline & & Ramp up & 0,005 \\
\hline & & Ramp down & 0,041 \\
\hline & High heeled & Up stairs & 0,048 \\
\hline & & Ramp down & 0,021 \\
\hline \multirow{14}{*}{$\begin{array}{l}\text { LERET - } \\
\text { support } \\
\text { RLL }\end{array}$} & Barefoot & Subir escada & 0,037 \\
\hline & & Down stairs & 0,002 \\
\hline & Slipper & Flat surface & $<0,001$ \\
\hline & & Up stairs & 0,015 \\
\hline & & Down stairs & 0,022 \\
\hline & & Ramp up & 0,004 \\
\hline & & Ramp down & 0,007 \\
\hline & Tennis shoe & Flat surface & $<0,001$ \\
\hline & & Down stairs & $<0,001$ \\
\hline & Small heeled & Ramp up & 0,011 \\
\hline & Salto alto & Flat surface & 0,026 \\
\hline & & Down stairs & 0,011 \\
\hline & & Ramp up & 0,022 \\
\hline & & Ramp down & $<0,001$ \\
\hline \multirow{17}{*}{$\begin{array}{l}\text { LERET - } \\
\text { support } \\
\text { LLL }\end{array}$} & Barefoot & Up stairs & 0,03 \\
\hline & & Down stairs & 0,003 \\
\hline & & Ramp down & 0,004 \\
\hline & Slipper & Flat surface & $<0,001$ \\
\hline & & Up stairs & 0,025 \\
\hline & & Down stairs & 0,036 \\
\hline & & Ramp up & 0,009 \\
\hline & & Ramp down & $<0,001$ \\
\hline & Tennis shoe & Flat surface & $<0,001$ \\
\hline & & Down stairs & $<0,001$ \\
\hline & & Ramp up & 0,003 \\
\hline & Small heeled & Down stairs & 0,005 \\
\hline & & Ramp up & 0,001 \\
\hline & & Ramp down & 0,039 \\
\hline & High heeled & Up stairs & 0,004 \\
\hline & & Ramp up & 0,004 \\
\hline & & Ramp down & 0,001 \\
\hline
\end{tabular}

*Right lower limb (RLL), Left lower limb (LLL)

\section{Discussion}

The results of electromyographyc analysis of the muscles evaluated showed that the muscles of the leg had greater electromyographyc activity in the position of pre-fatigue of the lower limbs and erector spinae in the post-fatigue of the lower limbs, in most of the conditions and situations.
The muscle TA is the primary engine for dorsiflexion of the ankle and aid in its stabilization [16].

In this study, the muscles LTA and RTA showed significant differences when the volunteers were barefoot in the conditions ramp up and ramp down, respectively, during the balance of the walk and with the small-heeled shoes, in the plan, during the balance of the walk. Moreover, there was also an increase in electromyographyc activity post-fatigue, except for the RTA barefoot ramp up.

The TA muscle controls the positioning of the foot both at the stage of support, with an increase in stability of the support of the segment in contact with the ground, as during the balance phase through the movement of dorsiflexion, which prepares the foot to the next support of the calcaneus, during the course of the walk $[17,18]$. This may be a factor that justified the difference found in those muscles while ramp up and down, during the balance phase of the walk.

Perry [18] describes a quick activation of the TA muscle during the initial phase of assessment of the march as a method of preparing for the detachment of the distal segment of the LL of the soil and in the terminal phase of the balance in order to maintain the dorsiflexion of the ankle to increase stability for the beginning of the new phase of the initial contact of the distal segment with double support.

With the co-activation of the muscles of the leg during the support of the distal segment of lower limb and because of the protocol of fatigue greater activation of the TA, can also manifest itself as a form of recruitment of this muscle for greater stability of the distal segment of lower limbs during the cycles of the march.

The muscle gastrocnemius is comprised of two portions, the medial and lateral. It is an important knee flexor and places the plantar flexion of the ankle, as a biarticular muscle.

On the activation of muscle RGM, there were significant differences when the volunteers were in slipper down stairs and tennis shoes in the plan, at the stage of support, and high-heeled shoes in the plan, during the balance of the march. There was also an increase in electromyographyc activity post-fatigue, only with the use of slipper.

The LGM showed significant differences on barefoot condition down stairs and ramp up at the stage of support from right lower limb of locomotion, with the use of tennis shoes while rising ladders at the stage of support of left lower limb, in the high-heeled shoes ramp down at the stage of support of left lower limb and climbing stairs at the stage of support of the 
right lower limb. There was also an increase of electromyographyc activity post-fatigue, only with the use of tennis shoes.

The RGL showed significant difference in barefoot condition in the flat surface, during the support of the march. The LGL showed significant difference in barefoot condition ramp up during the support of locomotion.

The gastrocnemius medial and lateral portion, aid in propulsion during the locomotion of the individual in daily and professional activities. The prevalence of major activity of this muscle during the support of the motion can be understood because the need of maintaining the stability of body mass, absorbing and dissipating the reactive forces, as both acts in the increase phase as the initial support to control the bending knee, as an biarticular muscle [19].

Gefen et al. [20] showed a significant increase in the GL electromyographyc activity in relation to GM with the use of high heels in women who had regular habit of use and non-regular. They inferred that lateral portion was more activated due to an intense need for contraction to compensate the strength of reaction soil. They also evaluated that the muscular fatigue of gastrocnemius was predominant in lateral portion during the use of high heeled shoe.

In this study, the medial portions of the muscle gastrocnemius had greater electromyographyc activity that the lateral portions. What can be explained by the fact the inversion movement of the feet increases with the imbalance body marked by the use of shoes with little stability as slippers and high heeled shoes and the low use of shoes with high heels $[16,20]$.

The ERET are important anti-gravity muscles and work predominantly on a static form during most of the day. Tissues liabilities, such as capsules, ligaments and discs of the spine become overwhelmed after the active elements become less effective and less on muscle fatigue [21].

Most of the conditions and situations before and after fatigue of the leg muscles assessed with the use of different shoes and barefoot, showed significant differences for the ERET muscles bilaterally.

The ERETE alone, showed significant differences only with the use of slipper upstairs at the stage of support from RLL and down stairs at the stage in support of the LLL locomotion. Using high heels showed significant differences down stairs, with support from RLL and the ramp up, with the support of RLL and LLL. The RERET isolated, had significant differences with the use of tennis, ramp up and down with support from RLL, and with small heels to upstairs with support from RLL.
No significant differences were found for ERET bilaterally on barefoot condition in the flat surface and to ramp up, with support from RLL and LLL, and ramp down with support from RLL, with the use of tennis, to upstairs, with support from RLL and LLL and ramp down with support from LLL, with the use of small heels in the flat surface, with support from RLL and LLL, upstairs with support of LLL, down stairs with support of RLL and ramp down with support of RLL; with the use of high heels, in support of the LLL, upstairs with support from RLL and down stairs with support of LLL.

For electromyographyc activity of the muscles of the erector spinae, significant difference in the condition of pre and post-fatigue in most of the conditions of study with all types of shoes and barefoot. These findings contradict with those found in the study of Bendix et al. [22] in which this muscles were evaluated and were not statistically significant differences with the use shoes to high heels.

The analysis indicated minor electromyographyc activity of the muscles of the spine when compared to the leg muscles in the position of pre-fatigue, due to lower demand of the voluntary muscle injury after reaching the required balance wearing high heeled shoes. Because of the use of the protocol of fatigue, it can be inferred that the erectors were more recruited to maintain the posture during locomotion, offsetting the reduction in the activation of other muscles evaluated. As suggested by Lee et al. [23], the erector muscles adapt to the situation of fatigue offsetting the reduction of activation of the muscles of lower limbs.

Casarin [24] found greater electromyographyc activity of the muscles GM and GL bilaterally than erectors spinae, so growing proportion to the elevation of the heel. There was no significant difference between the voluntary barefoot only on condition that the group was not using daily.

The slipper was the shoe that promoted greater electromyographyc activity of the erector spinae bilaterally. This may be justified by this be an unstable footwear without support on the side of the foot and the back of the calcaneus, when compared to other shoes used.

\subsection{Clinical Relevance}

The regular use of heels, especially the high, can lead to learning motor of the women that uses these shoes more often because the repetition of a task is a determining factor for cortical plasticity that, in turn, automatically generates the gesture [25]. Conse- 
quently, there may be a lower recruitment of fibers to perform a task and reduced energy expenditure.

The fatigue of the muscles of the leg has caused greater activation of the ERET muscles to compensate the reduction of activation of the fatigued muscles, which suggests a major energy expenditure of these muscles, with possible consequences functional.

Adaptive conditions, such as weakness, muscle imbalance and shortening associated with regular use of high shoes, has been widely questioned in relation to the incidence of injuries as sprains, knee and ankle, and postural changes such as increased lumbar lordosis or its rectification.

Therefore, preventive actions such as implementation of a programme of regular physical activity, muscle strengthening and stretching, should be prescribed and targeted to women with this habit, and should be implemented in companies in order to prevent injuries and tables of pain. Thus, it could prevent the decrease in productive capacity and decrease the rate of absenteeism, reducing expenses in the health system existing in the country.

\section{Conclusion}

The electromyographyc assessment showed, in most of the conditions, a greater activity of the muscles of the leg in pre-fatigue, particularly with highheeled shoes and slipper, probably by instability and non-habit of regular use of these shoes. In postfatigue of the lower limbs, there was a decline in the activities of the muscles of the leg and increased recruitment of the erector muscles justified by the need for greater stability of the spine and pelvis during the locomotion of young women.

\section{References}

[1] C. Frey, Foot Health and Shoewear for Women, Clinical Orthopaedics and related research. 372 (2000), 32-44.

[2] R. Snow and K. Williams, High heeled shoes: their effect on center of mass position, posture, three-dimensional kinematics, rear foot motion and ground reaction forces, Archives of Physical Medicine and Rehabilitation, 75 (1994),568-576.

[3] M. Esenyel, K. Walsh, J.G. Walden and A. Gitter, Kinetics of High-Heeled Gait. Journal of the American Podiatric Medical Association. 93 (2003), 27-32.

[4] W.H. Hong, Y. H. Lee, H.C. Chen, Y.C. Pei, C.Y. Wu, Influence of heel height and shoe insert on comfort perception and biomechanical performance of young female adults during walking, Foot \& Ankle International, 26 (2005), 1042-48.

[5] R. Neptune, F. Zajac and S. Kautz, Muscle force redistributes segmental power for body progression during walking, Gait \& Posture, 19 (2004), 194-205.
[6] M. Liu, F. Anderson, M. Pandy and S. Delp, Muscles that support the body also modulate forward progression during walking, Journal of Biomechanics, 39 (2006), 2623-2630.

[7] R. Neptune, S. Kautz and F. Zajac, Contributions of the individual ankle plantar flexors to support, forward progression and swing initiation during walking, Journal of Biomechanics, 34 (2001), 1387-1398.

[8] G. Cappellini, Y. Ivanenko, V. Poppele and F. Lacquaniti. Motor patterns in human walking and running, Journal of Neurophysiology. 95 (2006), 3426-3437.

[9] Grandjean E. Manual de Ergonomia: adaptando o trabalho ao homem, (4th ed), Porto Alegre: Bookman, 1998.

[10] J. Potério-Filho, S.A.F. Silveira, G.M.B. Potério, R.F. Junior, F.H.M. Almeida and F.H. Menezes. The effect of walking with high-heeled shoes on the leg venous pressure, Angiology, 57 (2006), 424-30.

[11] L. Anjos, Body mass index as a tool in the nutritional assessment of adults: a review. Revista de Saúde Pública, 26 (1992).

[12] Cram J, Kasman G, Holtz J. Introduction to Surface Electromyography. Aspen Publishers, Gaithersburg, Maryland, 1998.

[13] Fridlund AJ, Cacioppo JT. Guidelines for Human Electromyographic Research. Psycophysiology, 23(1986), 567-98.

[14] S. Silva and M. Gonçalves, Comparação de Protocolos para Verificação da Fadiga Muscular pela Eletromiografia de Superfície, Motriz, 9 (2003), 51-58.

[15] S. Silva and M. Gonçalves, Análise da fadiga muscular pela amplitude do sinal eletromiográfico, Revista Brasileira de Ciência e Movimento, 11 (2003), 15-20.

[16] P. Henderson and S. Piazza, A Biomechanical Evaluation of Standing in High-Heeled Shoes, [Acess in 07/06/08] Available in: www. gradsch.psu.edu.

[17] J. Li and Y. Hong, Kinematic and Electromyographic Analysis of the Trunk and Lower Limbs during Walking in Negative-Heeled Shoes, Journal of American Podiatric Medical Association, 97 (2007), 447-55.

[18] J. Perry, Análise de marcha. Barueri: Manole, 2005.

[19] D. Stefanyshyn, B. Nigg, V. Fisher, B. O'Flynn and W. Liu, The Influence of High Heeled Shoes on Kinematics, Kinetics, and Muscle EMG of Normal Female Gait, Journal of Applied Biomechanics, 16 (2000).

[20] A. Gefen, M. Megido-Ravid Y. Itzchak and M. Arcan, Analysis of muscular fatigue and foot stability during highheeled gait, Gait \& Posture, 15 (2000), 56-63.

[21] S. Roy, C. De Luca, M. Emley, L. Oddsson, R. Buijs, J. Levins $\mathrm{J}$ and et al., Classification of back muscle impairment based on the surface eletromyographic signal, Journal of Rehabilitation Research and Development, 34 (1997), 405-14.

[22] T. Bendix, S.S. Sorensen and K. Klausen, Lumbar Curve, Trunk Muscles and Line of Gravity with Different Heel Heights, Spine, 9 (1984), 223-27.

[23] K. Lee, J. Shieh, A. Matteliano and T. Smiehorowski, Electromyographic changes of leg muscles with heel lifts in women: therapeutic implications, Archives of Physical Medicine and Rehabilitation, 71 (1990), 31-33.

[24] C. Casarin, A influência do calçado de salto alto sobre a lordose lombar associada aos músculos lombares $e$ gastrocnêmio, 2005. 49 f. Tesis - Universidade Estadual de Campinas, Faculdade de Odontologia de Piracicaba. 2005.

[25] Anderson J. Cognitive Skills and Their Acquisition. Ed. Lawrence Erlbaum Associates, 1981. 